\title{
Algorithmic determination of immobile indices in convex SIP problems with polyhedral index sets
}

\author{
O. I. Kostyukova ${ }^{\mathrm{a}}$ and T. V. Tchemisova ${ }^{\mathrm{b}}$ \\ anstitute of Mathematics, National Academy of Sciences of Belarus, Surganov str. 11, \\ 220072, Minsk, Belarus; ${ }^{\mathrm{b}}$ Mathematical Department, University of Aveiro, Campus \\ Universitrio Santiago, 3810-193, Aveiro, Portugal
}

\section{ARTICLE HISTORY}

Compiled December 13, 2018

\begin{abstract}
The concepts of immobile indices and their immobility orders are objective and important characteristics of feasible sets of Semi-Infinite Programming (SIP) problems. They can be used for formulation of new efficient optimality conditions without constraint qualifications. Given a class of convex SIP problems with polyhedral index sets, we describe and justify a finite constructive algorithm (algorithm DIIPS) that allows to find in a finite number of steps all immobile indices and the corresponding immobility orders along the feasible directions. This algorithm is based on a representation of the cones of feasible directions in the polyhedral index sets in the form of linear combinations of extremal rays and on the approach described in our previous papers for the cases of immobile indices' sets of simpler structures. A constructive procedure of determination of the extremal rays is described and an example illustrating the application of the DIIPS algorithm is provided.
\end{abstract}

\section{KEYWORDS}

Semi-Infinite Programming (SIP), Convex Programming (CP), immobile index, immobility order, cone of feasible directions, extremal ray.

\section{AMS CLASSIFICATION}

90C25, 90C30, 90C34

\section{Introduction}

Semi-Infinite Programming (SIP) deals with extremal problems that involve infinitely many constraints in a finite dimensional space. Due to numerous theoretical and practical applications, today semi-infinite optimization is a topic of a special interest (see Bonnans and Shapiro (2000), Hettich and Kortanek (1993), López and Still (2007), Stein (2003), and the references therein). The most efficient methods for solving optimization problems are usually based on optimality conditions that permit not only to test the optimality of a given feasible solution, but also to find better directions to optimality. Usually the optimality conditions are formulated for certain classes of optimization problems. This allows for more effective use of specific structures of the problems under consideration (see e.g. Bonnans and Shapiro (2000), Stein (2003) et al.). 
In our papers (see e.g. Kostyukova et al. (2008), Kostyukova and Tchemisova (2014)) we introduce the concepts of immobile indices and their immobility orders for different classes of SIP problems. For a given a SIP problem, immobile indices can be defined as indices of the constraints that are active for all feasible solutions. It was shown that immobile indices are objective and important characteristics of feasible sets allowing to formulate efficient optimality conditions which do not use constraint qualifications (CQ) and can be successfully applied for building new constructive numerical methods. We proved optimality conditions for different classes of convex SIP problems, having formulated these conditions in both implicit and explicit form. Obviously, to use these optimality conditions, as well as to develop numerical methods based on these conditions, it is necessary to have practical algorithms that determine the immobile indices and their immobility orders. In our paper Kostyukova et al. (2008), we described such an algorithm for the case when the indices of the constraints have dimension one (the index set is a compact on the line). This algorithm cannot simply be generalized for the case of multidimensional index sets, because in this case the feasible sets have a more complex structure and, in turn, should be represented constructively in terms of their extremal rays.

In this paper, we describe and justify a finite constructive algorithm (algorithm $D I I P S)$ that determines immobile indices in convex SIP problems with polyhedral index sets. Given a feasible solution and the corresponding set of active indices, we describe the conforming cones of feasible directions in terms of the extremal rays. These rays are determined using a procedure that was specially elaborated for this purpose. Next, we use the DIIPS algorithm to find the set of immobile indices of the SIP problem and the corresponding immobility orders along the extremal directions. An example illustrating application of the DIIPS algorithm is provided.

\section{Immobile indices and immobility orders in SIP problems with polyhedral index sets}

Consider a convex Semi-Infinite Programming problem in the form

$$
\begin{array}{cc}
(P): & \min _{x \in \mathbb{R}^{n}} c(x) \\
\text { s.t. } & f(x, t) \leq 0 \quad \forall t \in T=\left\{t \in \mathbb{R}^{s}: h_{k}^{T} t \leq \Delta h_{k}, k \in K\right\},
\end{array}
$$

where $K$ is a finite index set; the objective function $c(x)$, and the constraint functions $f(x, t), t \in T$, are convex w.r.t. $x \in \mathbb{R}^{n}$; vectors $h_{k} \in \mathbb{R}^{s}$ and numbers $\Delta h_{k}, k \in K$ are given. Notice that here the index set $T$ is a convex polyhedron. Denote by $X$ the feasible set of problem $(P): X=\left\{x \in \mathbb{R}^{n}: f(x, t) \leq 0 \forall t \in T\right\}$.

Given $t \in T$, denote by $K_{a}(t) \subset K$ the corresponding set of active indices:

$$
K_{a}(t):=\left\{k \in K: h_{k}^{T} t=\Delta h_{k}\right\},
$$

and by $L(t)$ the corresponding set of feasible directions in $T$ :

$$
L(t):=\left\{l \in \mathbb{R}^{s}: h_{k}^{T} l \leq 0, k \in K_{a}(t)\right\} .
$$

Let $T_{a}(x) \subset T$ be the set of active in $x \in X$ indices: $T_{a}(x):=\{t \in T: f(x, t)=0\}$. 
Definition 2.1. An index $\bar{t} \in T$ is immobile in problem $(P)$ if

$$
f(x, \bar{t})=0 \text { for all } x \in X \text {. }
$$

Denote by $T^{*}$ the set of all immobile indices in $(P)$. It is evident that $T^{*} \subset T_{a}(x)$ for all $x \in X$.

Definition 2.2. The constraints of problem $(P)$ satisfy the Slater condition if there exists $\bar{x} \in \mathbb{R}^{n}$ such that $f(\bar{x}, t)<0, t \in T$.

In Kostyukova and Tchemisova (2012), it is proved that the convex SIP problem $(P)$ with $X \neq \emptyset$ satisfies the Slater condition (the Slater CQ) if and only if the set of immobile indices in this problem is empty. Therefore, the emptiness of the set $T^{*}$ can be considered as a constraint qualification which is equivalent to the Slater CQ.

The following definition determines important quantitative characteristics of the immobile indices.

Definition 2.3. Given an immobile index $\bar{t} \in T^{*}$ and a nontrivial feasible direction $\bar{l} \in L(\bar{t})$, let us say that $\bar{t}$ has the immobility order $q(\bar{t}, \bar{l})$ along $\bar{l}$ if

(1) $\left.\frac{d^{i} f(x, \bar{t}+\alpha \bar{l})}{d \alpha^{i}}\right|_{\alpha=+0}=0 \forall x \in X, i=0, \ldots, q(\bar{t}, \bar{l})$,

(2) there exists a feasible $\bar{x}=x(\bar{t}, \bar{l}) \in X$ such that $\left.\frac{d^{(q(\bar{t}, \bar{l})+1)} f(\bar{x}, \bar{t}+\alpha \bar{l})}{d \alpha^{(q(\bar{t}, l)+1)}}\right|_{\alpha=+0} \neq 0$.

Here and in what follows, we consider the set $\{i=s, \ldots, k\}$ to be empty if $k<s$. We denote $\left.\frac{d^{0} f(x, \bar{t}+\alpha \bar{l})}{d \alpha^{0}}\right|_{\alpha=+0}:=f(x, \bar{t})$.

Notice that Definition 2.3 can be easily generalized for all indices $t \in T$ if one sets $q(t, \bar{l}):=-1$ for any $t \in T \backslash T^{*}, \bar{l} \in L(t)$.

\section{The cone of feasible directions in the case of a polyhedral index set}

Given the convex SIP problem $(P)$, consider an index $\bar{t} \in T$. Denote by $\bar{L}:=L(\bar{t})$ the set of feasible in $\bar{t}$ directions that is defined in (3). Evidently, $\bar{L}$ is a polyhedral cone in $\mathbb{R}^{s}$ and hence it is finitely generated by some vector set in $\mathbb{R}^{s}$. In this section we will present constructive rules for finding this vector set.

\subsection{Representation of the set $\bar{L}$ in terms of the extremal rays}

Given $\bar{t} \in T$, consider the corresponding set $\bar{K}:=K_{a}(\bar{t})$ and the set $\Delta \bar{L} \subset \mathbb{R}^{s}$ defined as follows: $\Delta \bar{L}:=\left\{l \in \mathbb{R}^{s}: h_{k}^{T} l=0, k \in \bar{K}\right\}$. Evidently, $\Delta \bar{L}=\{\mathbf{0}\}$ for $m=s$ and $\Delta \bar{L}$ is a subspace of $\mathbb{R}^{s}$ for $m<s$, where $m=\operatorname{rank}\left(h_{k}, k \in \bar{K}\right)$.

Set $p:=s-m$ and suppose that the set

$$
\left\{b_{i}, i=1, \ldots, p\right\}
$$

is a basis of $\Delta \bar{L}$. Consider the set $\Delta \overline{\bar{L}}=\bar{L} \bigcap \Delta \bar{L}^{\perp}$, where $\Delta \bar{L}^{\perp}$ is the orthogonal complement of the subspace $\Delta \bar{L}$ in $\mathbb{R}^{s}$. One can easily prove that the set $\Delta \overline{\bar{L}}$ is a pointed cone, i.e. it is a cone with the following property:

$$
\text { for any } l \neq 0 \text { it holds: } l \in \Delta \overline{\bar{L}} \Rightarrow-l \notin \Delta \overline{\bar{L}} \text {. }
$$


Then there exists a finite set of vectors

$$
\left\{a_{i} \in \Delta \overline{\bar{L}}, i \in I\right\},
$$

such that the cone $\bar{L}$ can be represented in the form

$$
\bar{L}=\left\{l \in \mathbb{R}^{s}: l=\sum_{i=1}^{p} \beta_{i} b_{i}+\sum_{i \in I} \alpha_{i} a_{i}, \alpha_{i} \geq 0, i \in I\right\},
$$

where vectors $b_{i}, i \in\{1, \ldots, p\}$, are defined in (5) and $\beta_{i} \in \mathbb{R}, i \in\{1, \ldots, p\}$.

Therefore, for any $\bar{t} \in T$ there exist (finite) sets of vectors (5) and (6) such that the set of feasible directions in $\bar{t}$ can be represented in the form (7). The vectors of the sets (5), (6) are usually referred to as extremal rays, vectors (5) being bidirectional and vectors (6) being unidirectional rays.

\subsection{The rules for constructing the extremal rays}

In the literature, different methods for constructive representation of polyhedral cones are proposed (see for example, Chernikova (1968) and Fernandez and Quinton (1988)). Here we describe a simple procedure that can be used for the determination of the sets of extremal rays (5) and (6) and, therefore, for the representation of the set $\bar{L}$ in the form (7).

Given $k \in \bar{K}$, consider vector $h_{k}$ defining the index set of the problem $(P)$. Denote by $h_{k i}, i \in S:=\{1,2, \ldots, s\}$, the components of this vector: $h_{k}^{T}=\left(h_{k i}, i \in S\right)$. Let $H$ be a $|\bar{K}| \times|S|-$ matrix composed by these components:

$$
H=\left(\begin{array}{c}
h_{k i}, \quad i \in S \\
k \in \bar{K}
\end{array}\right)
$$

Consider subsets $S_{0} \subset S$ and $N_{0} \subset \bar{K}$ such that $\left|S_{0}\right|=\left|N_{0}\right|=m$ and the matrix

$$
H_{0}=H\left(N_{0}, S_{0}\right)=\left(\begin{array}{cc}
h_{k i}, & i \in S_{0} \\
k \in N_{0}
\end{array}\right)
$$

is not singular: $\operatorname{det}\left(H_{0}\right) \neq 0$. By construction, $H_{0}$ is a square sub-matrix of the matrix $H$ such that $\operatorname{rank} H=\operatorname{rank} H_{0}=m$.

Construct vectors

$$
\bar{b}_{i}=\left(\bar{b}_{i j}, j \in S\right), i \in S \backslash S_{0},
$$

whose components are defined by the following rules: $\bar{b}_{i j}=0, j \in S \backslash\left(S_{0} \bigcup i\right), b_{i i}=1$, $\left(\bar{b}_{i j}, j \in S_{0}\right)^{T}=-H_{0}^{-1}\left(\begin{array}{c}h_{k i} \\ k \in N_{0}\end{array}\right), i \in S \backslash S_{0}$.

It is easy to verify that vectors (9) form a basis of the space $\operatorname{Ker} H=\Delta \bar{L}$. Therefore we can set in (5) that $\left\{b_{i}, i=1, \ldots, p\right\}=\left\{\bar{b}_{i}, i \in S \backslash S_{0}\right\}$.

Consider vector $h_{0}:=\sum_{k \in \bar{K}} h_{k}$. If $h_{0}=\mathbf{0} \in \mathbb{R}^{s}$, then the set of vectors (6) in (7) is empty. 
Suppose that $h_{0} \neq \mathbf{0}$. Denote by $\Omega$ the set of subsets $N_{*} \subset \bar{K}$ such that $\left|N_{*}\right|=m-1$ and $\operatorname{det}\left(D\left(N_{*}\right)\right) \neq 0$, where $D\left(N_{*}\right)=\left(h_{0}, h_{k}, k \in N_{*} ; b_{i}, i=1, \ldots, p\right)^{T} \in \mathbb{R}^{s \times s}$.

Given $N_{*} \in \Omega$, let $a\left(N_{*}\right)$ be the first column of the matrix $-D^{-1}\left(N_{*}\right)$, i.e. $a\left(N_{*}\right)=-D^{-1}\left(N_{*}\right) e_{1}$. Set $\Omega_{*}:=\left\{N_{*} \in \Omega: h_{k}^{T} a\left(N_{*}\right) \leq 0, k \in \bar{K} \backslash N_{*}\right\}$. It can be easily verified that for representation (7) we can choose the set of vectors $a_{i}, i \in I$, in the form

$$
\left\{a_{i}, i \in I\right\}:=\left\{a\left(N_{*}\right), N_{*} \in \Omega_{*}\right\} .
$$

Remark 1. From the constructions above, it follows that in the case $m=\left|S_{0}\right|=|\bar{K}|$, we have $I=\{1, \ldots, m\}$, and the vectors $a_{i}=\left(a_{i j}, j \in S\right), i \in I$, can be constructed by the following rule: $a_{i j}=0, j \in S \backslash S_{0} ; \quad\left(a_{i j}, j \in S_{0}\right)^{T}=-H_{0}^{-1} e_{i}, i=1, \ldots, m$, where $e_{i} \in \mathbb{R}^{m}$ is the $i$-th vector of the canonic basis of $\mathbb{R}^{m}$, and the matrix $H_{0}$ is given in (8).

Remark 2. In the case $m=|S|=s$, the set $\left\{b_{i}, i=1, \ldots, p\right\}$ is empty since $p=0$.

Remark 3. As it was noted above, the set $\left\{a_{i}, i \in I\right\}$ is empty $(I=\emptyset)$ when $h_{0}=\mathbf{0}$. It can be proved that $h_{0} \neq \mathbf{0}$ if the interior of the polyhedral index set $T$ is not empty, i.e. if the constraints defining $T$ satisfy the Slater condition

$$
\exists \tilde{t} \in T: h_{k}^{T} \tilde{t}<\Delta h_{k} \forall k \in K .
$$

\section{Determination of the immobile indices and their immobility orders}

\subsection{Immobile indices and $C Q$-free optimality conditions}

Assumption 4.1. Suppose that given a convex SIP problem in the form $(P)$, it holds: $X \neq \emptyset$, the set $T$ is bounded, and

$$
q(t, l) \leq 1, \forall t \in T^{*}, \forall l \in L(t) \backslash\{\mathbf{0}\} .
$$

We consider here that conditions (11) are trivially fulfilled if $T^{*}=\emptyset$. $3.1)$.

The following result is proved in Kostyukova and Tchemisova (2014) (Proposition

Proposition 4.2. Assumption 4.1 implies that the set of immobile indices $T^{*}$ consists of a finite number of elements: $T^{*}=\left\{t_{j}^{*}, j \in J_{*}\right\}$ with some finite index set $J_{*}$, and there exists $\bar{x} \in X$ such that $\left|T_{a}(\bar{x})\right|<\infty$.

For any immobile index $t_{j}^{*}, j \in J_{*}$, consider the corresponding cone of feasible directions $L\left(t_{j}^{*}\right)$ defined in (3). Denote by

$$
b_{i}(j), i=1, \ldots, p_{j}, \quad a_{i}(j), i \in I(j), j \in J_{*},
$$

the extremal rays generating this cone. These rays can be found by the rules described in the previous section.

Denote

$$
I_{0}(j):=\left\{i \in I(j): q\left(t_{j}^{*}, a_{i}(j)\right)>0\right\}, I_{*}(j):=I(j) \backslash I_{0}(j) .
$$


It can be proved that under Assumption 4.1, the optimality conditions for problem $(P)$ have the form of the following criterion (Theorem 3.2 in Kostyukova and Tchemisova (2014)).

Theorem 4.3. Let Assumption 4.1 be fulfilled for the convex SIP problem $(P)$. Then a vector $x^{0} \in X$ is optimal in this problem if and only if there exists a finite set of indices $\left\{t_{j}, j \in J_{a}\left(x^{0}\right)\right\} \subset T_{a}\left(x^{0}\right) \backslash T^{*}$ where $\left|J_{a}\left(x^{0}\right)\right| \leq n$, such that the vector $x^{0}$ is optimal in the following auxiliary problem:

$$
\begin{gathered}
\min _{x \in \mathbb{R}^{n}} c(x) \\
\text { s.t. } \\
f\left(x, t_{j}\right) \leq 0, j \in J_{a}\left(x^{0}\right), \\
f\left(x, t_{j}^{*}\right)=0, \frac{\partial f^{T}\left(x, t_{j}^{*}\right)}{\partial t} B(j)=0, \frac{\partial f^{T}\left(x, t_{j}^{*}\right)}{\partial t} A_{0}(j)=0, \frac{\partial f^{T}\left(x, t_{j}^{*}\right)}{\partial t} A_{*}(j) \leq 0, \\
\left(\beta^{T}(j), \alpha_{0}^{T}(j)\right)\left(B(j), A_{0}(j)\right)^{T} \frac{\partial^{2} f\left(x, t_{j}^{*}\right)}{\partial t^{2}}\left(B(j), A_{0}(j)\right)\left(\begin{array}{c}
\beta(j) \\
\alpha_{0}(j)
\end{array}\right) \leq 0, \\
\forall\left(\beta(j), \alpha_{0}(j)\right) \in \mathbb{R}^{p(j)} \times \mathbb{R}_{+}^{\left|I_{0}(j)\right|}, j \in J_{*} .
\end{gathered}
$$

Here $\mathbb{R}_{+}^{m}:=\left\{t \in \mathbb{R}^{m}: t \geq 0\right\}$ and for every immobile index $t_{j}^{*} \in T^{*}, j \in J_{*}$ it holds

$$
B(j):=\left(b_{i}(j), i=1, \ldots, p(j)\right), A_{0}(j):=\left(a_{i}(j), i \in I_{0}(j)\right), A_{*}(j):=\left(a_{i}(j), i \in I_{*}(j)\right) .
$$

Theorem 4.3 gives optimality conditions for problem $(P)$ in the form of an implicit optimality criterion and uses the information about the immobile indices and the extremal rays representing the corresponding cones of feasible directions. In Kostyukova and Tchemisova (2014), these conditions were reformulated in different forms, including explicit optimality conditions. All these conditions are CQ-free and more efficient when compared with other optimality conditions known from the literature.

It is evident that to apply Theorem 4.3 one should know the set of immobile indices $T^{*}$ and the corresponding index sets (13). In the next section, we present a constructive algorithm for determination of the set of immobile indices and the corresponding sets (13) for problem $(P)$. We call this algorithm DIIPS since it Determines the Set of Immobile Indices in SIP problems with Polyhedral index Sets.

\subsection{Algorithm DIIPS}

Given a convex SIP problem in the form $(P)$, suppose that Assumption 4.1 is satisfied. It follows from Proposition 4.1 that there exists a feasible solution $\bar{x} \in X$ such that $T_{a}(\bar{x})=\left\{\bar{t}_{j}, j \in \bar{J}\right\}$ with $|\bar{J}|<\infty$. Suppose here that such a feasible solution $\bar{x} \in X$, the corresponding set $T_{a}(\bar{x})=\left\{\bar{t}_{j}, j \in \bar{J}\right\}$, the vectors $b_{i}(j), i=1, \ldots, p_{j} ; a_{i}(j), i \in I(j)$, defining the cones $L\left(\bar{t}_{j}\right), j \in \bar{J}$, and the index sets $\tilde{I}(j)=\left\{i \in I(j): \frac{\partial f^{T}\left(\bar{x}, \bar{t}_{j}\right)}{\partial t} a_{i}(j)=0\right\}, j \in \bar{J}$, are known.

Initializing. Set $J_{*}^{(0)}:=\emptyset$.

General iteration. We start the $(k+1)$-st iteration of the algorithm $(k \geq 0)$ having the following sets constructed on the previous iteration:

$$
J_{*}^{(k)} \subset \bar{J}, I_{0}^{(k)}(j) \subset \tilde{I}(j), j \in J_{*}^{(k)} .
$$


Notice that at the first iteration $(k=0)$ we do not use the sets $I_{0}^{(k)}(j) \subset \tilde{I}(j), j \in J_{*}^{(k)}$, since the set $J_{*}^{(0)}$ is empty.

Given $j \in J_{*}^{(k)}$, define

$$
L_{j}^{(k)}:=\left\{l \in \mathbb{R}^{s}: l=B(j) \beta_{j}+A_{0}^{(k)}(j) \alpha_{j}^{(k)}, \alpha_{j}^{(k)} \geq 0, l^{T} \frac{\partial^{2} f\left(\bar{x}, \bar{t}_{j}\right)}{\partial t^{2}} l=0,\|l\|=1\right\},
$$

where for $l \in \mathbb{R}^{s},\|l\|=\max _{i=1, \ldots, s}\left|l_{i}\right|$. Here $B(j):=\left(b_{i}(j), i=1, \ldots, p_{j}\right), \beta_{j} \in \mathbb{R}^{p_{j}}$ $A_{0}^{(k)}(j):=\left(a_{i}(j), i \in I_{0}^{(k)}(j)\right), \alpha_{j}^{(k)} \in \mathbb{R}_{+}^{\left|I_{0}^{(k)}(j)\right|}$.

Let us consider the following set:

$$
\begin{aligned}
& X^{(k+1)}:=\left\{x \in \mathbb{R}^{n}: f\left(x, \bar{t}_{j}\right) \leq 0, j \in \bar{J} \backslash J_{*}^{(k)} ; f\left(x, \bar{t}_{j}\right)=0, \frac{\partial f^{T}\left(x, \bar{t}_{j}\right)}{\partial t} b_{i}(j)=0, i=1, \ldots, p_{j} ;\right. \\
& \quad \frac{\partial f^{T}\left(x, \bar{t}_{j}\right)}{\partial t} a_{i}(j)\left\{\begin{array}{ll}
=0, & \text { for } i \in I_{0}^{(k)}(j) \\
\leq 0, & \text { for } i \in \tilde{I}(j) \backslash I_{0}^{(k)}(j)
\end{array}, l^{T} \frac{\partial^{2} f\left(x, \bar{t}_{j}\right)}{\partial t^{2}} l \leq 0, \forall l \in L_{j}^{(k)}, j \in J_{*}^{(k)}\right\} .
\end{aligned}
$$

It can be shown that $\bar{x} \in X^{(k+1)}$.

For all $j \in \bar{J} \backslash J_{*}^{(k)}$, consider an auxiliary problem

$$
\min f\left(x, \bar{t}_{j}\right), \quad \text { s.t. } x \in X^{(k+1)} .
$$

Set $x^{(j)}:=\bar{x}$ if $\bar{x}$ is optimal in the problem above; otherwise let $x^{(j)}$ be any vector satisfying the following conditions: $x^{(j)} \in X^{(k+1)}, f\left(x^{(j)}, \bar{t}_{j}\right)<0$.

Set $\Delta J_{*}^{(k+1)}:=\left\{j \in \bar{J} \backslash J_{*}^{(k)}: f\left(x^{(j)}, \bar{t}_{j}\right)=0\right\}$.

For all $i \in \tilde{I}(j) \backslash I_{0}^{(k)}(j), j \in J_{*}^{(k)}$, consider the following auxiliary problem:

$$
\min \frac{\partial f^{T}\left(x, \bar{t}_{j}\right)}{\partial t} a_{i}(j), \text { s.t. } x \in X^{(k+1)} .
$$

Let $x^{(i j)}:=\bar{x}$ if vector $\bar{x}$ is optimal in problem $(A u x(2))$, otherwise choose any vector $x^{(i j)} \in X^{(k+1)}$ such that $\frac{\partial f^{T}\left(x^{(i j)}, \bar{t}_{j}\right)}{\partial t} a_{i}(j)<0$.

Set $\Delta I_{0}^{(k+1)}(j):=\left\{i \in \tilde{I}(j) \backslash I_{0}^{(k)}(j): \frac{\partial f^{T}\left(x^{(i j)}, \bar{t}_{j}\right)}{\partial t} a_{i}(j)=0\right\}, j \in J_{*}^{(k)}$.

If $\Delta J_{*}^{(k+1)}=\emptyset$ and $\Delta I_{0}^{(k+1)}(j)=\emptyset \forall j \in J_{*}^{(k)}$, then the algorithm stops with

$$
T^{*}=\left\{t_{j}^{*}:=\bar{t}_{j}, j \in J_{*}:=J_{*}^{(k)}\right\},
$$

and

$q\left(t_{j}^{*}, a_{i}(j)\right)=1, j \in I_{0}(j):=I_{0}^{(k)}(j) ; \quad q\left(t_{j}^{*}, a_{i}(j)\right)=0, i \in I_{*}(j)=I(j) \backslash I_{0}^{(k)}(j), j \in J_{*}$.

Otherwise (if at least one of the sets $\Delta J_{*}^{(k+1)}$ and $\Delta I_{0}^{(k+1)}(j)$ is not empty), we set

$$
J_{*}^{(k+1)}:=J_{*}^{(k)} \bigcup \Delta J_{*}^{(k+1)}, I_{0}^{(k+1)}(j):=\left\{\begin{array}{c}
I_{0}^{(k)}(j) \bigcup \Delta I_{0}^{(k+1)}(j) \text { for } j \in J_{*}^{(k)} \\
\emptyset \text { for } j \in \Delta J_{*}^{(k+1)}
\end{array},\right.
$$


and proceed to the next iteration. The algorithm is described.

\subsection{Justification of the algorithm DIIPS}

To simplify the presentation, first suppose that we are applying the algorithm DIIPS to a linear w.r.t. $x$ SIP problem in the form $(P)$ that satisfies Assumption 4.1.

It is evident that the algorithm should stop after a finite number of iterations.

Suppose that the algorithm has stopped on the $(k+1)$-st iteration. Then we have the sets $J_{*}^{(k)} \subset \bar{J}, I_{0}^{(k)}(j) \subset \tilde{I}(j), j \in J_{*}^{(k)}$, and vectors $x^{(j)} \in X^{(k+1)}, j \in \bar{J} \backslash J_{*}^{(k)}$, $x^{(i j)} \in X^{(k+1)}, i \in \tilde{I}(j) \backslash I_{0}^{(k)}(j), j \in J_{*}^{(k)}$ such that

$$
f\left(x^{(j)}, \bar{t}_{j}\right)<0, j \in \bar{J} \backslash J_{*}^{(k)}, \quad \frac{\partial f^{T}\left(x^{(i j)}, \bar{t}_{j}\right)}{\partial t} a_{i}(j)<0, i \in \tilde{I}(j) \backslash I_{0}^{(k)}(j), j \in J_{*}^{(k)} .
$$

Since the function $f(x, t)$ is supposed to be linear w.r.t. $x$ and the set $X^{(k+1)}$ is convex, then there exists $\hat{x} \in X^{(k+1)}$ such that

$$
f\left(\hat{x}, \bar{t}_{j}\right)<0, j \in \bar{J} \backslash J_{*}^{(k)}, \frac{\partial f^{T}\left(\hat{x}, \bar{t}_{j}\right)}{\partial t} a_{i}(j)<0, i \in \tilde{I}(j) \backslash I_{0}^{(k)}(j), j \in J_{*}^{(k)} .
$$

It follows from the algorithm DIIPS that $\left\{\bar{t}_{j}, j \in J_{*}^{(k)}\right\} \subset T^{*}$ and $q\left(\bar{t}_{j}, l\right)>0$ for

$$
l=B_{j} \beta_{j}+A_{0 j}^{(k)} \alpha_{0 j}^{(k)}=\left(B_{j}, A_{0 j}^{(k)}\right)\left(\begin{array}{c}
\beta_{j} \\
\alpha_{0 j}^{(k)}
\end{array}\right) \neq \mathbf{0}, \alpha_{0 j}^{(k)} \geq 0, j \in J_{*}^{(k)},
$$

where $A_{0 j}^{(k)}=\left(a_{i}(j), i \in I_{0}^{(k)}(j)\right), \alpha_{0 j}^{(k)} \in \mathbb{R}^{\left|I_{0}^{(k)}(j)\right|}, B_{j}=\left(b_{i}(j), i=1, \ldots, p_{j}\right), \beta_{j} \in \mathbb{R}^{p_{j}}$.

Hence from Assumption 4.1, it follows

$$
q\left(l, \bar{t}_{j}\right)=1, \text { for } l=\left(B_{j}, A_{0 j}^{(k)}\right)\left(\begin{array}{c}
\beta_{j} \\
\alpha_{0 j}^{(k)}
\end{array}\right) \neq \mathbf{0}, \alpha_{0 j}^{(k)} \geq 0, j \in J_{*}^{(k)} .
$$

Lemma 4.4. Let Assumption 4.1 be fulfilled and $j \in J_{*}^{(k)}$. Then there exists $x^{* j} \in X$ such that

$$
(A \tilde{\beta})^{T} \frac{\partial^{2} f\left(x^{* j}, \bar{t}_{j}\right)}{\partial t^{2}} A \tilde{\beta}<0 \text { for all } \tilde{\beta}=\tilde{\beta}(j)=\left(\beta_{j}, \alpha_{0 j}^{(k)}\right)^{T}, \alpha_{0 j}^{(k)} \geq 0,\|\tilde{\beta}\|=1,
$$

where $\mathcal{A}=\mathcal{A}(j):=\left(B_{j}, A_{0 j}^{(k)}\right)$.

Proof. Define the following function:

$$
F(x, \tilde{\beta}):=(A \tilde{\beta})^{T} \frac{\partial^{2} f\left(x, \bar{t}_{j}\right)}{\partial t^{2}} A \tilde{\beta} \leq 0, \tilde{\beta} \in \mathbf{B}, x \in X
$$

where $\mathbf{B}:=\left\{\tilde{\beta}=\left(\beta_{j}, \alpha_{0 j}^{(k)}\right)^{T}, \alpha_{0 j}^{(k)} \geq 0,\|\tilde{\beta}\|=1\right\}$. 
By construction, $X \subset \mathbb{R}^{n}$ is a convex set, $\mathbf{B}$ is compact, and the function $F(x, \tilde{\beta})$ is convex w.r.t. $x$. Consider any set of vectors

$$
\left\{\tilde{\beta}^{r}: \tilde{\beta}^{r} \in \mathbf{B}, r=1, \ldots, n+1\right\} .
$$

According to $(16)$ we have $q\left(\bar{t}_{j}, l\right)=1$, for all $l=A \tilde{\beta} \neq \mathbf{0}, \tilde{\beta} \in \mathbf{B}$. Then, by Definition 2.3 , for each $w=1, \ldots, n+1$, there exists $x^{(w)} \in X$, satisfying the following inequality:

$$
F\left(x^{(w)}, \tilde{\beta}^{w}\right)<0
$$

From the condition $x^{(w)} \in X$, it follows that $F\left(x^{(w)}, \tilde{\beta}^{r}\right) \leq 0 \forall r \neq w, r=1, \ldots, n+1$. Set $\overline{\bar{x}}:=\frac{1}{n+1} \sum_{\omega=1}^{n+1} x^{(\omega)}$. It is easy to check that

$$
\overline{\bar{x}} \in X \text { and } F\left(\overline{\bar{x}}, \tilde{\beta}^{r}\right)<0, \forall r=1, \ldots, n+1 .
$$

Thus we have showed that for any set (19) there exists the vector $\overline{\bar{x}}$ satisfying (20). Then, according to Proposition 3 from Kostyukova and Tchemisova (2012), for the given $j \in J_{*}^{(k)}$, there exists $x^{* j} \in X$ such that $F\left(x^{* j}, \tilde{\beta}\right)<0, \forall \tilde{\beta} \in \mathbf{B}$, i.e. inequalities (17) take place.

Set $\tilde{x}^{*}:=\sum_{j \in J_{*}^{(k)}} \frac{x^{* j}}{\left|J_{*}^{(k)}\right|}$, where $x^{* j} \in X, j \in J_{*}^{(k)}$, are the vectors considered in Lemma 4.4. Then $\tilde{x}^{*}$ satisfies the following conditions:

$$
\tilde{x}^{*} \in X, \quad l^{T} \frac{\partial^{2} f\left(\tilde{x}^{*}, \bar{t}_{j}\right)}{\partial t^{2}} l<0 \forall l=B_{i} \beta_{j}+A_{0 j}^{(k)} \alpha_{0 j}^{(k)} \neq \mathbf{0}, \alpha_{0 j}^{(k)} \geq 0, j \in J_{*}^{(k)} .
$$

Moreover, we know that given an immobile index $\bar{t}_{j}, j \in J_{*}=J_{*}^{(k)}$, for any $x \in X$, it holds

$$
f\left(x, \bar{t}_{j}\right)=0, \frac{\partial f^{T}\left(x, \bar{t}_{j}\right)}{\partial t} b_{i}(j)=0, i=1, \ldots, p_{j} ; \quad \frac{\partial f^{T}\left(x, \bar{t}_{j}\right)}{\partial t} a_{i}(j)=0, i \in I_{0}^{(k)}(j), j \in J_{*}^{(k)} .
$$

Then, evidently, for the constructed above vector $\tilde{x}^{*}$, we have

$$
\begin{gathered}
f\left(\tilde{x}^{*}, \bar{t}_{j}\right)=0, \quad \frac{\partial f^{T}\left(\tilde{x}^{*}, \bar{t}_{j}\right)}{\partial t} b_{i}(j)=0, i=1, \ldots, p_{j} \\
\frac{\partial f^{T}\left(\tilde{x}^{*}, \bar{t}_{j}\right)}{\partial t} a_{i}(j)=0, i \in I_{0}^{(k)}(j), \quad \frac{\partial f^{T}\left(\tilde{x}^{*}, \bar{t}_{j}\right)}{\partial t} a_{i}(j) \leq 0, i \in I(j) \backslash I_{0}^{(k)}(j), j \in J_{*}^{(k)} .
\end{gathered}
$$

Let $z:=\frac{1}{2}\left(\tilde{x}^{*}+\bar{x}\right) \in X$, where $\bar{x}$ is the vector introduced in section 4.1. Then, by construction, the following relations are satisfied: 


$$
\begin{aligned}
& f\left(z, \bar{t}_{j}\right) \leq 0, j \in \bar{J} \backslash J_{*}^{(k)}, \quad f\left(z, \bar{t}_{j}\right)=0, j \in J_{*}^{(k)} ; \\
& \frac{\partial f^{T}\left(z, \bar{t}_{j}\right)}{\partial t} b_{i}(j)=0, i=1, \ldots, p_{j} ; \quad \frac{\partial f^{T}\left(z, \bar{t}_{j}\right)}{\partial t} a_{i}(j)\left\{\begin{array}{c}
<0, \quad i \in I(j) \backslash \tilde{I}(j) \\
\leq 0, \quad i \in \tilde{I}(j) ;
\end{array}\right. \\
& l^{T} \frac{\partial^{2} f\left(z, \bar{t}_{j}\right)}{\partial t} l<0 \forall l \in L^{0 k}\left(\bar{t}_{j}\right) ; \quad l^{T} \frac{\partial^{2} f\left(z, \bar{t}_{j}\right)}{\partial t} l \leq 0 \forall l \in L\left(z, \bar{t}_{j}\right), \quad j \in J_{*}^{(k)},
\end{aligned}
$$

where

$$
\begin{aligned}
& L^{0 k}\left(\bar{t}_{j}\right):=\left\{l=B_{i} \beta_{i}+A_{0 i}^{(k)} \alpha_{0 i}^{(k)}, \alpha_{0 i}^{(k)} \geq 0,\left(\beta_{i}, \alpha_{0 i}^{(k)}\right) \neq \mathbf{0}\right\} \\
& L\left(z, \bar{t}_{j}\right):=\left\{l=B_{i} \beta_{i}+A_{i} \alpha_{i}, \alpha_{i} \geq 0, \frac{\partial f^{T}\left(z, \bar{t}_{j}\right)}{\partial t} l=0\right\}, j \in J_{*}^{(k)} .
\end{aligned}
$$

Given $\lambda \in[0,1]$, let us consider vector $x(\lambda):=(1-\lambda) z+\lambda \hat{x}$, where vector $\hat{x} \in X^{(k+1)}$ satisfies (15). Taking into account the linearity of $f(x, t)$ w.r.t. $x$, we have

$$
f\left(x(\lambda), \bar{t}_{j}\right)=(1-\lambda) f\left(z, \bar{t}_{j}\right)+\lambda f\left(\hat{x}, \bar{t}_{j}\right) .
$$

Then we can conclude that for $0<\lambda<1$, it holds

$$
\begin{aligned}
& f\left(x(\lambda), \bar{t}_{j}\right)<0 \text { for } j \in \bar{J} \backslash J_{*}^{(k)} ; f\left(x(\lambda), \bar{t}_{j}\right)=0 \text { for } j \in J_{*}^{(k)} ; \\
& \frac{\partial f^{T}\left(x(\lambda), \bar{t}_{j}\right)}{\partial t} b_{i}(j)=0, i=1, \ldots, p_{j} ; \\
& \frac{\partial f^{T}\left(x(\lambda), \bar{t}_{j}\right)}{\partial t} a_{i}(j)=0, i \in I_{0}^{(k)}(j), \frac{\partial f^{T}\left(x(\lambda), \bar{t}_{j}\right)}{\partial t} a_{i}(j)<0, i \in I(j) \backslash I_{0}^{(k)}(j) ; \\
& l^{T} \frac{\partial^{2} f\left(x(\lambda), \bar{t}_{j}\right)}{\partial t^{2}} l<0 \forall l \in L^{0 k}\left(\bar{t}_{j}\right), j \in J_{*}^{(k)} .
\end{aligned}
$$

It is evident that for a sufficiently small $\lambda>0$, we can guarantee that there exists $\varepsilon(\lambda) \geq 0$ such that $\varepsilon(\lambda) \rightarrow 0$ as $\lambda \rightarrow 0$ and

$$
f(x(\lambda), t)<0, t \in T \backslash \bigcup_{j \in J_{*}^{(k)}} T_{\varepsilon(\lambda)}\left(\bar{t}_{j}\right),
$$

where $T_{\varepsilon}(t)=\{\tau \in T:\|t-\tau\| \leq \varepsilon\}$.

Suppose that $j \in J_{*}^{(k)}$. Then any $t \in T_{\varepsilon(\lambda)}\left(\bar{t}_{i}\right)$ can be presented in the form $t=$ $\bar{t}_{j}+\Delta t_{j}, \Delta t_{j} \in L\left(\bar{t}_{j}\right),\left\|\Delta t_{j}\right\| \leq \varepsilon(\lambda)$ and in a rather small neighborhood of $t$ the 
following asymptotic expansion holds:

$$
\begin{aligned}
f(x(\lambda), t)= & f\left(x(\lambda), \bar{t}_{j}+\Delta t_{j}\right) \\
= & f\left(x(\lambda), \bar{t}_{j}\right)+\frac{\partial f^{T}\left(x(\lambda), \bar{t}_{j}\right)}{\partial t} \Delta t_{j}+\frac{1}{2} \Delta t_{j}^{T} \frac{\partial^{2} f\left(x(\lambda), \bar{t}_{j}\right)}{\partial t^{2}} \Delta t_{j}+o\left(\left\|\Delta t_{j}\right\|^{2}\right) \\
= & \sum_{i \in I(j) \backslash I_{0}^{(k)}(j)} \frac{\partial f^{T}\left(x(\lambda), \bar{t}_{j}\right)}{\partial t} a_{i}(j) \alpha_{i}(j) \\
& +\frac{1}{2}\left(\beta_{j}, \alpha_{j}\right)^{T}\left(B_{j}, A_{j}\right)^{T} \frac{\partial^{2} f\left(x(\lambda), \bar{t}_{j}\right)}{\partial t^{2}}\left(B_{j}, A_{j}\right)\left(\begin{array}{c}
\beta_{j} \\
\alpha_{j}
\end{array}\right)+o\left(\left\|\left(\beta_{j}, \alpha_{j}\right)\right\|^{2}\right),
\end{aligned}
$$

where $\alpha_{j}=\left(\alpha_{i}(j), i \in I(j)\right) \geq 0$.

Notice here that if $\left(\alpha_{i}(j), i \in I(j) \backslash I_{0}^{(k)}(j)\right) \neq \mathbf{0}$, then the first-order term in the expansion above is negative. If $\left(\alpha_{i}(j), i \in I(j) \backslash I_{0}^{(k)}(j)\right)=\mathbf{0}$, then this term vanishes and we get

$f(x(\lambda), t)=\left(\beta_{j}, \alpha_{0 j}^{(k)}\right)^{T}\left(B_{j}, A_{0 j}^{(k)}\right)^{T} \frac{\partial^{2} f\left(x(\lambda), \bar{t}_{j}\right)}{\partial t^{2}}\left(B_{j}, A_{0 j}^{(k)}\right)\left(\begin{array}{c}\beta_{j} \\ \alpha_{0 j}^{(k)}\end{array}\right)+o\left(\left\|\left(\beta_{j}, \alpha_{0 j}^{(k)}\right)\right\|^{2}\right)$.

In this case, $f(x(\lambda), t)<0$ when $\left(\beta_{j}, \alpha_{0 j}^{(k)}\right) \neq \mathbf{0}$ (taking into account (21)), and $f(x(\lambda), t)=f\left(x(\lambda), \bar{t}_{j}\right)=0$ when $\left(\beta_{j}, \alpha_{0 j}^{(k)}\right)=\mathbf{0}$. Then

$$
f(x(\lambda), t)<0, t \in T_{\varepsilon(\lambda)}\left(\bar{t}_{j}\right) \backslash \bar{t}_{j}, j \in J_{*}^{(k)},
$$

for sufficiently small $\lambda>0$.

Therefore, it is proved that for a sufficiently small $\lambda>0$ the vector $\tilde{x}=x(\lambda)$ has the following properties:

(P1) $\tilde{x} \in X$, i.e. $\tilde{x}$ is a feasible solution of problem (P) (it follows from (22), (23)).

(P2) The following relations are valid:

$$
\begin{aligned}
& f\left(\tilde{x}, \bar{t}_{j}\right)=0, \frac{\partial f^{T}\left(\tilde{x}, \bar{t}_{j}\right)}{\partial t} b_{i}(j)=0, i=1, \ldots, p_{j} ; \\
& \frac{\partial f^{T}\left(\tilde{x}, \bar{t}_{j}\right)}{\partial t} a_{j}(j)=0, i \in I_{0}^{(k)}(j), \frac{\partial f^{T}\left(\tilde{x}, \bar{t}_{j}\right)}{\partial t} a_{i}(j)<0, i \in I(j) \backslash I_{0}^{(k)}(j) ; \\
& l^{T} \frac{\partial^{2} f\left(\tilde{x}, \bar{t}_{j}\right)}{\partial t^{2}} l<0 \forall l \in L^{0 k}\left(\bar{t}_{j}\right), j \in J_{*}^{(k)} ; \\
& f(\tilde{x}, t)<0, \quad t \in T \backslash\left\{\bar{t}_{j}, j \in J_{*}^{(k)}\right\} .
\end{aligned}
$$

Recall that by construction, $\left\{\bar{t}_{j}, j \in J_{*}^{(k)}\right\} \subset T^{*}, I_{0}^{(k)}(j) \subset I_{0}(j), j \in J_{*}^{(k)}$. Then, taking into account Definition 2.3, we can conclude that

$$
T^{*}=\left\{\bar{t}_{j}, j \in J_{*}^{(k)}\right\}, I_{0}(j)=I_{0}^{(k)}(j), \forall j \in J_{*}^{(k)},
$$

and the algorithm DIIPS is justified.

In the case of convex (w.r.t. $x$ ) constraint functions, the steps of the algorithm are the same. To justify the algorithm in this case, one can use the same scheme as above, 
taking into account Lemmas 1-4 from Kostyukova and Tchemisova (2015).

Lemma 4.5. In Assumption 4.1, condition (11) is equivalent to the following statement: for any immobile index $\bar{t} \in T^{*}$, there exists $\bar{x}=\bar{x}(\bar{t}) \in X$ such that vector $\bar{t}$ satisfies the sufficient conditions of strict local maximum in the problem

$$
\max f(\bar{x}, t) \text {, s.t. } t \in T \text {, }
$$

and these conditions have the form

$$
\begin{aligned}
& \exists y_{k} \geq 0, k \in \bar{K}=\left\{k \in K: h_{k}^{T} \bar{t}=\Delta h_{k}\right\} \quad \text { such that } \frac{\partial f(\bar{x}, \bar{t})}{\partial t}=\sum_{k \in \bar{K}} h_{k} y_{k}, \\
& l^{T} \frac{\partial^{2} f(\bar{x}, \bar{t})}{\partial t^{2}} l<0 \forall l \in L(\bar{t}, \bar{x}):=\left\{l \in \mathbb{R}^{s}: l \neq \boldsymbol{O}, \frac{\partial f^{T}(\bar{x}, \bar{t})}{\partial t} l=0, h_{k}^{T} l \leq 0, k \in \bar{K}\right\} .
\end{aligned}
$$

Proof. Suppose that Assumption 4.1 is satisfied. It was proved above that there exists a vector $\tilde{x}$ that satisfies properties (P1) and (P2). Hence for any $\bar{t} \in T^{*}$ we can choose the vector $\bar{x}=\bar{x}(\bar{t})=\tilde{x}$.

Now let us consider a situation when for some $\bar{t} \in T^{*}$ there exists a vector $\bar{x} \in X$ satisfying (24). If suppose that condition (11) is not satisfied, then we get that there exists $\bar{l} \in L(\bar{t}), \bar{l} \neq \mathbf{0}$ such that $q(\bar{t}, \bar{l})>1$. Then from the definition of the immobility order it follows that

$$
\frac{\partial f^{T}(x, \bar{t})}{\partial t} \bar{l}=0, \bar{l}^{T} \frac{\partial^{2} f(x, \bar{t})}{\partial t} \bar{l}=0 \forall x \in X
$$

These equalities with $x=\bar{x} \in X$ contradict (24), and the lemma is proved.

\subsection{Remarks}

- It is evident that if the constraint function $f(x, t)$ is linear w.r.t. $x$, then the corresponding auxiliary problems $(\operatorname{Aux}(1))$ and $(\operatorname{Aux}(2))$ are linear w.r.t. $x$. On the base of Lemmas 1-4 from Kostyukova and Tchemisova (2015), one can prove that in the case of a convex w.r.t. $x$ constraint function $f(x, t)$, the auxiliary problems $(\operatorname{Aux}(1))$ and $(\operatorname{Aux}(2))$ are also convex w.r.t. $x$.

- On the iterations of the algorithm, we do not need to solve the auxiliary problems $(\operatorname{Aux}(1))$ and $(\operatorname{Aux}(2))$. We only check the optimality of the given feasible solution $\bar{x}$ in each of these problems and in the case when the solution is not optimal, find a feasible solution with a better (smaller) value of the cost function. Notice that this better solution is needed only for the justification of the algorithm.

- Often the sets $L_{j}^{(k)}, j \in J_{*}^{(k)}$, are either empty or consist of a finite number of elements. In these cases, in each of two problems $(\operatorname{Aux}(1))$ and $(\operatorname{Aux}(2))$, there is a finite number of constraints. In the general case, when a set $L_{j}^{(k)}$ consists of an infinite number of elements (it can be the union of a finite number of polyhedrons), at least one of the corresponding problem $(\operatorname{Aux}(1))$ or $(\operatorname{Aux}(2))$ possesses an infinite number of constraints

$$
g(x, l):=l^{T} \frac{\partial^{2} f\left(x, \bar{t}_{j}\right)}{\partial t^{2}} l \leq 0 \forall l \in L_{j}^{(k)}
$$


But we should notice that these constraints are simpler than the original constraints $f(x, t) \leq 0$ since the function $g(x, l)$ is quadratic w.r.t. the index variable $l$, while the dependence of the function $f(x, t)$ on the index variable $t$, as a rule, is more complex. The case when $f(x, t)$ depends linearly on $t$, is not of particular interest since then the original SIP problem (1) can be reduced to a convex programming problem (with a finite number of constraints).

\section{Example}

Consider the following SIP problem:

$$
\begin{gathered}
\min -x_{2}+x_{3}, \\
\text { s.t. } \\
f_{1}(x, t) \leq 0, \forall t \in T_{1}, \quad f_{2}(x, t) \leq 0, \forall t \in T_{2},
\end{gathered}
$$

where $x=\left(x_{1}, x_{2}, x_{3}, x_{4}\right)^{T} \in \mathbb{R}^{4}, t=\left(t_{1}, t_{2}\right)^{T} \in \mathbb{R}^{2}$, and

$$
\begin{aligned}
& f_{1}(x, t)=-t_{1}^{2} x_{1}+t_{1} t_{2} x_{1}+t_{1} x_{2}+\left(\sin t_{1}\right) x_{3}+t_{1} x_{4}-t_{2}^{2}, \\
& f_{2}(x, t)=t_{2} x_{1}+\left(t_{2}+1\right)^{2} x_{2}+\left(1-t_{2}\right) x_{3}+x_{4}-\left(t_{1}-3\right)^{2}+\left(t_{1}-3\right) t_{2} ; \\
& T_{1}=\left\{t \in \mathbb{R}^{2}:-t_{1}+t_{2} \leq 0, t_{1} \leq 2,-1 \leq t_{2}\right\}, \\
& T_{2}=\left\{t \in \mathbb{R}^{2}: t_{1}-t_{2} \leq 3,2 \leq t_{1} \leq 4,0 \leq t_{2} \leq 2\right\} .
\end{aligned}
$$

The index set here has the form $T=T_{1} \cup T_{2}$, where the sets $T_{1}$ and $T_{2}$ are polyhedrons which can be represented as follows:

$$
\begin{aligned}
& T_{1}=\left\{t \in \mathbb{R}^{2}: h_{1}^{T} t \leq 0, h_{2}^{T} t \leq 2, h_{3}^{T} t \leq 1\right\}, \\
& T_{2}=\left\{t \in \mathbb{R}^{2}: g_{1}^{T} t \leq 3, g_{2}^{T} t \leq 4, g_{3}^{T} t \leq-2, g_{4}^{T} t \leq 2, g_{5}^{T} t \leq 0\right\},
\end{aligned}
$$

if we suppose that $h_{1}^{T}=(-1,1), h_{2}^{T}=(1,0), h_{3}^{T}=(0,-1), g_{1}^{T}=(1,-1), g_{2}^{T}=(1,0)$, $g_{3}^{T}=(-1,0), g_{4}^{T}=(0,1), g_{5}^{T}=(0,-1)$.

Problem (26) admits a feasible solution $\bar{x}=\left(\bar{x}_{1}, \bar{x}_{2}, \bar{x}_{3}, \bar{x}_{4}\right)^{T}$, where

$$
\begin{gathered}
\bar{x}_{1}=\frac{\sin 2+2}{2} \approx 1.455, \quad \bar{x}_{2}=\frac{\left(\bar{x}_{1}\right)^{2}+\bar{x}_{1}(\sin 2-6)}{-2(\sin 2-2)} \approx-2.425, \\
\bar{x}_{3}=\bar{x}_{1}+2 \bar{x}_{2}, \quad \bar{x}_{4}=-3 \bar{x}_{2}-\bar{x}_{1} .
\end{gathered}
$$

It can be shown that the active index set at $\bar{x}$ has the form $T_{a}(\bar{x})=\left\{t^{(1)}, t^{(2)}, t^{(3)}\right\}$ with $t^{(1)}:=(0,0)^{T} \in T_{1}, t^{(2)}:=(3,0)^{T} \in T_{2}, t^{(3)}:=\left(2, \bar{x}_{1}\right)^{T} \in T_{1}$, and

$$
f_{1}\left(\bar{x}, t^{(1)}\right)=f_{2}\left(\bar{x}, t^{(2)}\right)=f_{1}\left(\bar{x}, t^{(3)}\right)=0 .
$$

Let us describe the cones of feasible directions $\bar{L}\left(t^{(i)}\right), t^{(i)} \in T_{a}(\bar{x}), i=1,2,3$, (see (3)) in the form (7) using the rules from section 3.

Consider, first, the active index $t^{(1)}=(0,0)^{T}$. Since $K_{a}\left(t^{(1)}\right)=\{1\}$ and $h_{1}=$ $(-1,1)^{T}$, we conclude that $\bar{L}_{1}=\bar{L}\left(t^{(1)}\right)=\left\{\left(l_{1}, l_{2}\right)^{T}:-l_{1}+l_{2} \leq 0\right\}$. 
In this example $s=2$, and hence $S=\{1,2\}$. Since $K_{a}\left(t^{(1)}\right)=\{1\}$, the corresponding matrix $H$ has the form $H=\left[h_{11}=-1, h_{12}=1\right]$. Having supposed $S_{0}=\{1\}, N_{0}=\{1\}$, we get $H_{0}=H\left(N_{0}, S_{0}\right)=[-1]$ and $H_{0}^{-1}=[-1]$.

Taking into account that $S \backslash S_{0}=\{2\}$, we can find the components of the bidirectional extremal ray $b(1)$ corresponding to $t^{(1)}: \bar{b}_{22}=1$ and $\bar{b}_{21}=-H_{0}^{-1} h_{12}=1$. Then $b(1)=(1,1)^{T}$. Now, let us find the unidirectional rays corresponding to $t^{(1)}$. Consider vector $h_{0}=(-1,1)^{T} \neq \mathbf{0}$. Since $m=1$, we get that $\left|N_{*}\right|=m-1=0$ and hence $N_{*}=\emptyset$. Then the matrix $D\left(N_{*}\right)$ has the form $D\left(N_{*}\right)=\left(\begin{array}{rr}-1 & 1 \\ 1 & 1\end{array}\right), D\left(N_{*}\right)^{-1}=\left(\begin{array}{rr}-\frac{1}{2} & \frac{1}{2} \\ \frac{1}{2} & \frac{1}{2}\end{array}\right)$, and $a\left(N_{*}\right)=-\left(\begin{array}{r}-\frac{1}{2} \\ \frac{1}{2}\end{array}\right), h_{1}^{T} a\left(N_{*}\right)=(-1,1)\left(\begin{array}{c}\frac{1}{2} \\ -\frac{1}{2}\end{array}\right)=-1 \leq 0$, and $a(1)=\left(\frac{1}{2},-\frac{1}{2}\right)^{T}$.

Therefore we conclude that the set $\bar{L}_{1}$ has two extremal rays, $b(1)=(1,1)^{T}$ and $a(1)=\left(\frac{1}{2},-\frac{1}{2}\right)^{T}$.

In an analogous way, we can show that the extremal rays of the set

$$
\bar{L}_{2}=\bar{L}\left(t^{(2)}\right)=\left\{\left(l_{1}, l_{2}\right): l_{1} \leq l_{2}, l_{2} \geq 0\right\}
$$

have the form $a_{1}(2)=(1,1)^{T}, a_{2}(2)=(-1,0)^{T}$, and the extremal rays of the set $\bar{L}_{3}=\bar{L}\left(t^{(3)}\right)=\left\{\left(l_{1}, l_{2}\right): l_{1} \leq 0\right\}$ have the form $a(3)=(-1,0)^{T}$ and $b(3)=(0,1)^{T}$.

Hence in this example we have the following extremal rays: $a(1)=(1,-1)^{T}, b(1)=$ $(1,1)^{T}, a_{1}(2)=(1,1)^{T}, a_{2}(2)=(-1,0)^{T}$, and $a(3)=(-1,0)^{T}, b(3)=(0,1)^{T}$.

Now we will apply the algorithm DIIPS and determine the immobile indices and their immobility orders in the problem from the example. Notice that

$$
\frac{\partial f_{1}^{T}\left(\bar{x}, t^{(1)}\right)}{\partial t} a(1)=0, \frac{\partial f_{2}^{T}\left(\bar{x}, t^{(2)}\right)}{\partial t} a_{1}(2)=0, \frac{\partial f_{2}^{T}\left(\bar{x}, t^{(2)}\right)}{\partial t} a_{2}(2)=0, \frac{\partial f_{1}^{T}\left(\bar{x}, t^{(3)}\right)}{\partial t} a(3) \neq 0 .
$$

Using the same notations as in section 4.2, we consider the following sets:

$$
\bar{J}=\{1,2,3\}, \tilde{I}(1)=\{1\}, \tilde{I}(2)=\{1,2\}, \tilde{I}(3)=\emptyset .
$$

On the first iteration of the algorithm set $k=0$. Then $J_{*}^{(0)}=\emptyset$ and

$$
\begin{aligned}
X^{(1)} & =\left\{x \in \mathbb{R}^{n}: f_{1}\left(x, t^{(1)}\right) \leq 0, f_{2}\left(x, t^{(2)}\right) \leq 0, f_{1}\left(x, t^{(3)}\right) \leq 0\right\} \\
& =\left\{x \in \mathbb{R}^{4}: x_{2}+x_{3}+x_{4} \leq 0,\left(2 \bar{x}_{1}-4\right) x_{1}+2 x_{2}+\sin 2 \cdot x_{3}+2 x_{4}-\left(\bar{x}_{1}\right)^{2} \leq 0\right\} .
\end{aligned}
$$

Consider the auxiliary problem $(A u x(1))$ for each $j \in \bar{J}=\{1,2,3\}$. When $j=1$, this problem has the form

$$
\min _{x \in X^{(1)}} f_{1}\left(x, t^{(1)}\right)
$$

Since for each $x \in X^{(1)}$ it holds $f_{1}\left(x, t^{(1)}\right)=0$, we can set $x^{(1)}=\bar{x}$.

Let $j=2$. In this case the problem $(A u x(1))$ takes the form

$$
\min _{x \in X^{(1)}} f_{2}\left(x, t^{(2)}\right) .
$$


Since the objective function of this problem, $f_{2}\left(x, t^{(2)}\right)=x_{2}+x_{3}+x_{4}$, is not bounded from below, then according to the algorithm, we can choose any feasible solution $x$ such that $f_{2}\left(x, t^{(2)}\right)<0$. Choose $x^{(2)}=(0,0,0,-1)^{T}$ such that $f_{2}\left(x^{(2)}, t^{(2)}\right)=-1<0$. The same situation occurs for $j=3$ : the objective function of the problem

$$
\min _{x \in X^{(1)}} f_{1}\left(x, t^{(3)}\right), \quad \text { with } f_{1}\left(x, t^{(3)}\right)=\left(2 \bar{x}_{1}-4\right) x_{1}+2 x_{2}+\sin 2 \cdot x_{3}+2 x_{4}-\left(\bar{x}_{1}\right)^{2},
$$

is not bounded from below, and we can set $x^{(3)}=(0,0,0,0)^{T}$ since $f_{1}\left(x^{(3)}, t^{(3)}\right)=$ $-\left(\bar{x}_{1}\right)^{2}<0$.

Find the sets $\Delta J_{*}^{(1)}:=\left\{i \in \bar{J}: f\left(x^{(i)}, t^{(i)}\right)=0\right\}=\{1\}, \Delta I_{0}^{(1)}(1):=\emptyset$.

Since $\Delta J_{*}^{(1)}=\{1\} \neq \emptyset$, we proceed to the next iteration with

$$
J_{*}^{(1)}=J_{*}^{(0)} \bigcup \Delta J_{*}^{(1)}=\{1\}, I_{0}^{(1)}(1)=\Delta I_{0}^{(1)}(1)=\emptyset \text { and } \bar{J} \backslash J_{*}^{(1)}=\{2,3\} .
$$

On the next iteration $(k=1)$ we consider the set

$$
\begin{gathered}
X^{(2)}=\left\{x \in \mathbb{R}^{4}: f_{2}\left(x, t^{(2)}\right) \leq 0, f_{1}\left(x, t^{(3)}\right) \leq 0, f_{1}\left(x, t^{(1)}\right)=0, \frac{\partial f_{1}^{T}\left(x, t^{(1)}\right)}{\partial t} b(1)=0,\right. \\
\left.\frac{\partial f_{1}^{T}\left(x, t^{(1)}\right)}{\partial t} a(1) \leq 0, l^{T} \frac{\partial^{2} f_{1}\left(x, t^{(1)}\right)}{\partial t^{2}} l \leq 0, l \in L_{1}^{(1)}\right\},
\end{gathered}
$$

where the set $L_{1}^{(1)}$ is defined as in (14) for $J_{*}^{(1)}=\{1\}: L_{1}^{(1)}=\emptyset$ since $l^{t} \frac{\partial^{2} f_{1}\left(\bar{x}, t^{(1)}\right)}{\partial t^{2}} l<0$ for all $l \in \mathbb{R}^{2} \backslash\{0\}$. Then

$$
X^{(2)}=\left\{x \in \mathbb{R}^{4}: x_{2}+x_{3}+x_{4}=0,\left(2 \bar{x}_{1}-4\right) x_{1}+2 x_{2}+\sin 2 \cdot x_{3}+2 x_{4}-\left(\bar{x}_{1}\right)^{2} \leq 0\right\} .
$$

For $j=2$, the auxiliary problem $(A u x(1))$ has the form

$$
\min _{x \in X^{(2)}} f_{2}\left(x, t^{(2)}\right)
$$

Since $f_{2}\left(x, t^{(2)}\right)=x_{2}+x_{3}+x_{4}=0 \forall x \in X^{(2)}$, we can set $x^{(2)}=\bar{x}$.

For $j=3$, the problem $(\operatorname{Aux}(1))$ takes the form

$$
\min _{x \in X^{(2)}} f_{1}\left(x, t^{(3)}\right) \text { with } f_{1}\left(x, t^{(3)}\right)=\left(2 \bar{x}_{1}-4\right) x_{1}+2 x_{2}+\sin 2 \cdot x_{3}+2 x_{4}-\left(\bar{x}_{1}\right)^{2},
$$

and it easy to conclude that the objective function of this problem is not bounded from below. Then we can choose $x^{(3)}=(0,0,0,0)^{T}$, as $f_{1}\left(x^{(3)}, t^{(3)}\right)=-\left(\bar{x}_{1}\right)^{2}<0$.

Construct the set $\Delta J_{*}^{(2)}=\left\{i \in\{2,3\}: f\left(x^{(i)}, t^{(i)}\right)=0\right\}=\{2\}$.

Since $\tilde{I}(1) \backslash I_{0}^{(1)}(1)=\{1\}$, we have to consider the auxiliary problem $(A u x(2))$

$$
\min _{x \in X^{(2)}} \frac{\partial f_{1}^{T}\left(x, t^{(1)}\right)}{\partial t} a(1)
$$

Since $\frac{\partial f_{1}^{T}\left(x, t^{(1)}\right)}{\partial t} a(1)=x_{2}+x_{3}+x_{4}$, the objective function of this problem is equal to zero for all feasible solutions and therefore we can choose $x^{(11)}=\bar{x}$. 
According to the DIIPS algorithm,

$$
\Delta I_{0}^{(2)}(1)=\left\{j \in\{1\}: \frac{\partial f_{1}^{T}\left(x^{(1)}, t^{(1)}\right)}{\partial t} a(1)=0\right\}=\{1\} \neq \emptyset .
$$

Construct the sets

$$
J_{*}^{(2)}=J_{*}^{(1)} \bigcup \Delta J_{*}^{(2)}=\{1,2\}, I_{0}^{(2)}(1)=I_{0}^{(1)}(1) \bigcup \Delta I_{0}^{(2)}(2)=\{1\}, I_{0}^{(2)}(2)=\emptyset
$$

and proceed to the next iteration.

For $k=2$, we construct the set

$$
\begin{gathered}
X^{(3)}=\left\{x \in \mathbb{R}^{4}: f_{1}\left(x, t^{(3)}\right) \leq 0, f_{1}\left(x, t^{(1)}\right)=0, f_{2}\left(x, t^{(2)}\right)=0,\right. \\
\frac{\partial f_{1}^{T}\left(x, t^{(1)}\right)}{\partial t} b(1)=0, \frac{\partial f_{1}^{T}\left(x, t^{(1)}\right)}{\partial t} a(1)=0, l^{T} \frac{\partial^{2} f_{1}\left(x, t^{(1)}\right)}{\partial t^{2}} l \leq 0, l \in L_{1}^{(2)}, \\
\left.\frac{\partial f_{2}^{T}\left(x, t^{(2)}\right)}{\partial t} a_{1}(2) \leq 0, \frac{\partial f_{2}^{T}\left(x, t^{(2)}\right)}{\partial t} a_{2}(2) \leq 0, \quad l^{T} \frac{\partial^{2} f_{2}\left(x, t^{(2)}\right)}{\partial t^{2}} l \leq 0, \quad l \in L_{2}^{(2)}\right\},
\end{gathered}
$$

where $L_{1}^{(2)}$ and $L_{1}^{(2)}$ are defined in (14). Since $l^{T} \frac{\partial^{2} f_{i}\left(\bar{x}, t^{(i)}\right)}{\partial t^{2}} l<0$ for all $l \in \mathbb{R}^{2} \backslash\{\mathbf{0}\}$, $i=1,2$, we have $L_{1}^{(2)}=L_{2}^{(2)}=\emptyset$. Substituting the functions and simplifying the expression, we get

$$
\begin{gathered}
X^{(3)}=\left\{x \in \mathbb{R}^{4}:\left(2 \bar{x}_{1}-4\right) x_{1}+2 x_{2}+\sin 2 \cdot x_{3}+2 x_{4}-\left(\bar{x}_{1}\right)^{2} \leq 0\right. \\
\left.x_{2}+x_{3}+x_{4}=0, x_{1}+2 x_{2}-x_{3} \leq 0\right\}
\end{gathered}
$$

Then the problem $(\operatorname{Aux}(1))$ takes the form

$$
\min _{x \in X^{(3)}} f_{1}\left(x, t^{(3)}\right)
$$

or explicitly

$$
\begin{array}{ll} 
& \min \left(2 \bar{x}_{1}-4\right) x_{1}+2 x_{2}+\sin 2 \cdot x_{3}+2 x_{4}-\left(\bar{x}_{1}\right)^{2} \\
\text { s.t. } & x_{2}+x_{3}+x_{4}=0, x_{1}+2 x_{2}-x_{3} \leq 0, \\
& -4 x_{1}+2 \bar{x}_{1} x_{1}+2 x_{2}+\sin 2 \cdot x_{3}+2 x_{4}-\left(\bar{x}_{1}\right)^{2} \leq 0 .
\end{array}
$$

The objective function is not bounded from below. Choose $x^{(3)}=(0,0,0,0)^{T}$ with $f_{1}\left(x^{(3)}, t^{(3)}\right)<0$ and construct $\left.\Delta J_{*}^{(3)}=\left\{i \in\{3\}: f_{1}\left(x^{(i)}\right), t^{(i)}\right)=0\right\}=\emptyset$.

For $j \in J_{*}^{(2)}=\{1,2\}$, consider the sets $\tilde{I}(j) \backslash I_{0}^{(2)}(j)$ :

$$
\tilde{I}(1) \backslash I_{0}^{(2)}(1)=\emptyset, \tilde{I}(2) \backslash I_{0}^{(2)}(2)=\tilde{I}(2)=\{1,2\} .
$$

For $i \in \tilde{I}(2) \backslash I_{0}^{(2)}(2)=\{1,2\}$, the corresponding auxiliary problems $($ Aux $(2))$ take the forms

$$
\min _{x \in X^{(3)}} \frac{\partial^{T} f_{2}\left(x, t^{(2)}\right)}{\partial t} a_{1}(2), \text { and } \min _{x \in X^{(3)}} \frac{\partial^{T} f_{2}\left(x, t^{(2)}\right)}{\partial t} a_{2}(2)
$$


or equivalently,

$$
(A): \quad \min _{x \in X^{(3)}} x_{1}+2 x_{2}-x_{3}, \quad \text { and }(B): \min _{x \in X^{(3)}} 0 .
$$

The problem $(A)$ can be rewritten in the form

$$
\begin{array}{ll} 
& \min x_{1}+2 x_{2}-x_{3}, \\
\text { s.t. } & x_{2}+x_{3}+x_{4}=0, x_{1}+2 x_{2}-x_{3} \leq 0,(\sin 2-2) x_{3}-\left(\bar{x}_{1}\right)^{2} \leq 0 .
\end{array}
$$

Since the objective function of this problem is not bounded from below on the set $X^{(3)}$, we choose $x^{(21)}=(0,-2,0,2)^{T}$ such that $f_{2}\left(x^{(21)}, t^{(2)}\right)=-4$.

Notice here that in the auxiliary problem $(B)$, the value of the objective function is constant and equal to zero. Hence we can consider any feasible solution as the optimal and set, for example, $x^{(22)}=\bar{x}$.

Then $\Delta I_{0}^{(3)}(1)=\emptyset$ and $\Delta I_{0}^{(3)}(2)=\left\{i \in\{1,2\}: \frac{\partial f_{2}^{T}\left(x^{(2 i)}, t^{(2)}\right)}{\partial t} a_{i}(2)=0\right\}=\{2\}$.

Here we have $\Delta J_{*}^{(3)}=\emptyset, \Delta I_{0}^{(3)}(1)=\emptyset$, but $\Delta I_{0}^{(3)}(2) \neq \emptyset$, and, therefore, we pass to the next iteration, with $k=3$, the sets

$$
\begin{gathered}
J_{*}^{(3)}=J_{*}^{(2)} \bigcup \Delta J_{*}^{(3)}=\{1,2\}, I_{0}^{(3)}(1)=I_{0}^{(2)}(1) \bigcup \Delta I_{0}^{(3)}(1)=\{1\}, \\
I_{0}^{(3)}(2)=I_{0}^{(2)}(2) \bigcup \Delta I_{0}^{(3)}(2)=\{2\},
\end{gathered}
$$

and

$$
\begin{aligned}
& X^{(4)}=\left\{x \in \mathbb{R}^{4}: f_{1}\left(x, t^{(3)}\right) \leq 0, f_{1}\left(x, t^{(1)}\right)=0, f_{2}\left(x, t^{(2)}\right)=0,\right. \\
& \frac{\partial f_{1}^{T}\left(x, t^{(1)}\right)}{\partial t} b(1)=0, \frac{\partial f_{1}^{T}\left(x, t^{(1)}\right)}{\partial t} a(1)=0, l^{T} \frac{\partial^{2} f_{1}\left(x, t^{(1)}\right)}{\partial t^{2}} l \leq 0, l \in L_{1}^{(3)}, \\
& \left.\frac{\partial f_{2}^{T}\left(x, t^{(2)}\right)}{\partial t} a_{1}(2) \leq 0, \frac{\partial f_{2}^{T}\left(x, t^{(2)}\right)}{\partial t} a_{2}(2)=0, l^{T} \frac{\partial^{2} f_{2}\left(x, t^{(2)}\right)}{\partial t^{2}} l \leq 0, l \in L_{2}^{(3)}\right\} .
\end{aligned}
$$

Having substituted the explicit representations of the sets and functions involved in the description of the set $X^{(4)}$, and having simplified the obtained expressions, we get

$$
\begin{gathered}
X^{(4)}=\left\{x \in \mathbb{R}^{4}:\left(2 \bar{x}_{1}-4\right) x_{1}+2 x_{2}+\sin 2 \cdot x_{3}+2 x_{4}-\left(\bar{x}_{1}\right)^{2} \leq 0,\right. \\
\left.x_{2}+x_{3}+x_{4}=0, x_{1}+2 x_{2}-x_{3} \leq 0\right\} .
\end{gathered}
$$

Then, the auxiliary problem $(A u x(1))$ takes the form

$$
\min _{x \in X^{(4)}}\left(2 \bar{x}_{1}-4\right) x_{1}+2 x_{2}+\sin 2 \cdot x_{3}+2 x_{4}-\left(\bar{x}_{1}\right)^{2} .
$$

The objective function of this problem is not bounded from below, therefore we can choose $x^{(3)}=(0,0,0,0)^{T}$. Then $f_{1}\left(x^{(3)}, t^{(3)}\right)=-\left(\bar{x}_{1}\right)^{2}<0, \Delta J_{*}^{(4)}=\emptyset$.

For $i \in \tilde{I}(2) \backslash I_{0}^{(3)}(2)=\{1\}$, the auxiliary problem $(A u x(2))$ has the form

$$
\min _{x \in X^{(4)}} x_{1}+2 x_{2}-x_{3}
$$

and its objective function is not bounded from below on the set $X^{(4)}$. For $x^{(21)}=$ $(0,-2,0,2)^{T} \in X^{(4)}$ we have $f_{2}\left(x^{(21)}, t^{(2)}\right)=-4<0$. Hence $\Delta I_{0}^{(4)}(2)=\emptyset$. Since $\Delta I_{0}^{(4)}(2)=\emptyset, \Delta J_{*}^{(4)}=\emptyset$, the algorithm stops with $T^{*}=\left\{t^{(j)}, j \in J_{*}^{(3)}\right\}=\left\{t^{(1)}, t^{(2)}\right\}$. 
The immobility orders of the immobile indices along the extremal rays are as follows:

$$
q\left(t^{j}, a_{i}(j)\right)=1, i \in I_{0}^{(3)}(j) ; \quad q\left(t^{j}, a_{i}(j)\right)=0, i \in \tilde{I}(i) \backslash I_{0}^{(3)}(j), j \in J_{*}^{(3)} .
$$

Hence we conclude that

$$
q\left(t^{(1)}, a(1)\right)=1, q\left(t^{(1)}, b(1)\right)=1, q\left(t^{(2)}, a_{2}(2)\right)=1, q\left(t^{(2)}, a_{1}(2)\right)=0 .
$$

Let us show that there exists a vector $\tilde{x}$ satisfying properties (P1) and (P2). We will search for this vector in the form $\tilde{x}=x(\lambda)=(1-\lambda) \bar{x}+\lambda \hat{x}$, for some $\lambda \in[0,1]$. Let us set $\hat{x}:=\frac{1}{2}\left(x^{(3)}+x^{(21)}\right)=(0,-1,0,1)^{T}$.

Due to linearity of functions $f_{i}(x, t), i=1,2$, w.r.t. $x$ we have

$$
f_{i}(x(\lambda), t)=(1-\lambda) f_{i}(\bar{x}, t)+\lambda f_{i}(\hat{x}, t), i=1,2 .
$$

It is easy to check that

$$
\begin{gathered}
f_{1}(\hat{x}, t)=-t_{2}^{2} \Longrightarrow f_{1}(\hat{x}, t)<0 \text { for all } t=\left(t_{1}, t_{2}\right), t_{2} \neq \mathbf{0} ; \\
f_{2}(\hat{x}, t)=-t_{2}^{2}+t_{1} t_{2}-t_{1}^{2}+6 t_{1}-5 t_{2}-9 \Longrightarrow f_{2}(\hat{x}, t)<0 \text { for all } t=\left(t_{1}, t_{2}\right) \in T_{2} \backslash\left\{t^{(2)}\right\} .
\end{gathered}
$$

Taking into account that

$$
f_{1}(\bar{x}, t)<0 \text { for all } t \in T_{1} \backslash\left\{t^{(1)}, t^{(3)}\right\}, \quad f_{2}(\bar{x}, t)<0 \text { for all } t \in T_{2} \backslash\left\{t^{(2)}\right\},
$$

we conclude that

$f_{1}(x(\lambda), t)<0$ for all $t \in T_{1} \backslash\left\{t^{(1)}\right\}, \quad f_{2}(x, t)<0$ for all $t \in T_{2} \backslash\left\{t^{(2)}\right\}, \quad \forall \lambda \in(0,1)$.

It is easy to check that $\frac{\partial f_{2}^{T}\left(x(\lambda), t^{(2)}\right)}{\partial t} a_{1}(2)=-2 \lambda$ and $\frac{\partial f_{2}^{T}\left(x(\lambda), t^{(2)}\right)}{\partial t} a_{1}(2)<0 \forall \lambda \in(0,1)$. Notice that $L_{j}^{(3)}=\emptyset, j \in J_{*}^{(3)}$. Hence we have shown that all vectors $x(\lambda), \lambda \in(0,1)$, satisfy properties (P1) and (P2).

If we set $\lambda:=\frac{1}{2}$, we get $\tilde{x}=\frac{1}{2} \bar{x}+\frac{1}{2} \hat{x}=\left(\tilde{x_{1}}, \tilde{x_{2}}, \tilde{x_{3}}, \tilde{x_{4}}\right)^{T}$, where by construction, $\tilde{x_{1}}=\frac{1}{2} \bar{x}_{1}, \tilde{x_{2}}=\frac{1}{2}\left(\bar{x}_{2}-1\right), \tilde{x_{3}}=\frac{1}{2} \bar{x}_{3}$, and $\tilde{x_{4}}=\frac{1}{2}\left(\bar{x}_{4}+1\right)$.

\section{Conclusions}

The main contribution of the paper consist in the following: we have proposed a simple constructive procedure for the determination of the extremal rays of polyhedral cones, described and justified the algorithm DIIPS for the determination of the immobile indices and their immobility orders in convex SIP problems with polyhedral index sets. These results can be easily implemented by different numerical procedures.

The algorithm DIIPS presented in the paper has several important applications:

- it can be applied for the determination of the immobile indices and therefore, for verifying the Slater constraint qualification;

- it may be used for formulation and testing the CQ-free optimality conditions for different classes of convex SIP problems which can be described in terms of the SIP problems with polyhedral index sets (for example, semi-infinite polynomial programming problems, copositive programming problems); 
- it can be applied in presolving procedures during numerical solving of convex SIP problems.

The main steps of the algorithm can be reformulated for more general classes of problems, such as, for example, SIP problems with finitely represented index sets. In this case some substantial modifications of the algorithm should be done and this is the purpose of our future work.

\section{Acknowledgements}

This work was partially supported by the state research program "Convergence"(Republic of Belarus), Task 1.3.01 and by Portuguese funds through CIDMA - Center for Research and Development in Mathematics and Applications, and FCT - Portuguese Foundation for Science and Technology, within the project UID/MAT/04106/2013.

The authors thank the anonymous referees for their helpful comments and corrections that permitted to improve the presentation of this paper.

\section{References}

Bonnans JF, Shapiro A. 2000. Perturbation Analysis of Optimization Problems. SpringerVerlag, New-York.

Chernikova NV. 1968. An algorithm for discovering the set of all the solutions of a linear programming problem. Comp. Math. Math. Phys. 8(6): 282-293. In Russian.

Fernandez F, Quinton P. 1988. Extension of Chernikova's algorithm for solving general mixed linear programming problems, INRIA-Unité de Recherche INRIA-RENNES- Rapport de recherche n 943, programme 5 .

Hettich R, Kortanek KO. 1993. Semi-infinite Programming: theory, methods and applications, SIAM Rev. 35: 380-429.

Kostyukova OI, Tchemisova TV. 2012. Implicit optimality criterion for convex SIP problem with box constrained index set. TOP, 20(2): 475-502.

Kostyukova OI, Tchemisova TV. 2014. On a constructive approach to optimality conditions for convex SIP problems with polyhedral index sets, Optimization, 63(1): 67-91.

Kostyukova OI, Tchemisova TV. 2015. Convex SIP problems with finitely representable compact index sets: immobile indices and the properties of the auxiliary NLP problem, SetValued Var. Anal., 23 (3): 519-546.

Kostyukova OI, Tchemisova TV, Yermalinskaya SA. 2008. On the algorithm of determination of immobile indices for convex SIP problems, IJAMAS-International Journal on Mathematics and Statistics 13, J08: 13-33.

López M, Still G. 2007. Semi-Infinite Programming. EJOR 180(2): 491-518.

Stein O, Still G. 2000. On optimality conditions for generalized Semi-infinite Programming problems. J. Optim. Theory Appl. 104(2):443-458.

Stein O. 2003. Bi-level strategies in Semi-infinite Programming. Kluwer, Boston. 